histopathological findings, light, electron microscopy, and immunofluorescence (IF) for deposition of $\operatorname{IgM}$, IgG, IgA, $\mathrm{C} 1 \mathrm{q}$, and $\mathrm{C} 3$ was determined in 23 of the 33 patients for whom renal biopsy data was available.

Results LN was identified in $41 \%$ of our cohort of 80 SLE patients. Naïve B-cell IFN $\beta$ was positively associated with the development of LN but not cutaneous disease. Higher levels of B-cell IFN $\beta$ also correlated with higher levels of circulating anti-dsDNA, anti-Sm, and the urinary protein/creatinine ratio. Biopsy examination revealed that proliferative LN lesions (Class III, IV with or without V) characterized by significantly elevated endocapillary hypercellularity, fibrous crescent, and fibrocellular crescent were significantly associated with high Bcell IFN $\beta$. Surprisingly, IgG, IgA, IgM, C3, and C1q deposition in the kidney was not correlated with B-cell IFN $\beta$.

Conclusions Our results suggest that B-cell IFN $\beta$ can be used in combination with other clinical diagnostic markers to assist in identifying patients who are at high risk of developing advanced LN.

Acknowledgments This work was supported by the VA Merit Review grant [I01BX004049]; the NIH grants [R01-AI071110, R01 AI134023], the Lupus Research Alliance Distinguished Innovator Award; the LRA Target Identification in Lupus Award; and to support flow cytometry analysis [P30AR-048311 and P30-AI-027767]. Funders had no role in design, analysis, and reporting.

\section{DISEASE FLARES IN LUPUS ARE CONCORDANT WITH RUMINOCOCCUS BLAUTIA GNAVUS BLOOMS ARISING WITHIN UNSTABLE GUT MICROBIOTA COMMUNITIES}

${ }^{1}$ Doua F Azzouz, 'Ze Chen, ${ }^{1}$ Peter Izmirly, ${ }^{2}$ David Fenyo, ${ }^{1}$ jill Buyon, ${ }^{3}$ Alexander V Alekseyenko, 'Gregg J Silverman*. 'Department of Medicine, NYU Grossman School of Medicine, New York; ${ }^{2}$ Institute for Systems Genetics, NYU Grossman School of Medicine, New York; ${ }^{3}$ Biomedical Informatics Center, MUSC Charleston, 29203

\subsection{6/lupus-2021-lupus21century.29}

Background Whereas genetic susceptibility for the development of the archetypic autoimmune disease, Systemic Lupus Erythematosus, has been well explored the precipitants for clinical disease flares remain a mystery. We have therefore investigated for dynamic time-dependent relationships between gut-microbial communities and Lupus disease activity.

Methods Patients fullfilled ACR criteria and were not treated with recent antibiotics or cytotoxic agents. Disease activity defined by modified SLEDAI, with fecal $16 \mathrm{~S}$ rRNA libraries generated, and data were analyzed as previously described. Taxonomic surveys were performed on sequential 16S rRNA gene amplicon-libraries from 16 individual female Lupus patients and 22 healthy volunteers from fecal samples obtained at serial timepoints, over many months to several years.

Results Lupus patients commonly displayed significant imbalances in alpha and beta microbiota-diversity, and patients were uniquely different from the healthy individuals as well as different from other Lupus patients - a pattern of disease-associated heterogeneity in dysbiotic communities termed the Anna Karenina Principle. From community-wide ecological multivariate analysis of sequential libraries obtained overtime, patients displayed significantly greater longitudinal microbiota community instability that was most exaggerated in Lupus Nephritis patients. Furthermore, taxonomic analyses documented an absence of bacterial intestinal blooms in 9 healthy adults with up to 12 serial fecal $16 \mathrm{~S}$ libraries. In contrast, many of the microbiota communities of 16 Lupus patients demonstrated transient spikes of pathogenic bacterial species, with by far the most prevalent were blooms of Ruminococcus blautia gnavus (RG), which were documented in 4/9 Lupus Nephritis and 1/7 non-renal patients, concordant with disease flares. Importantly,

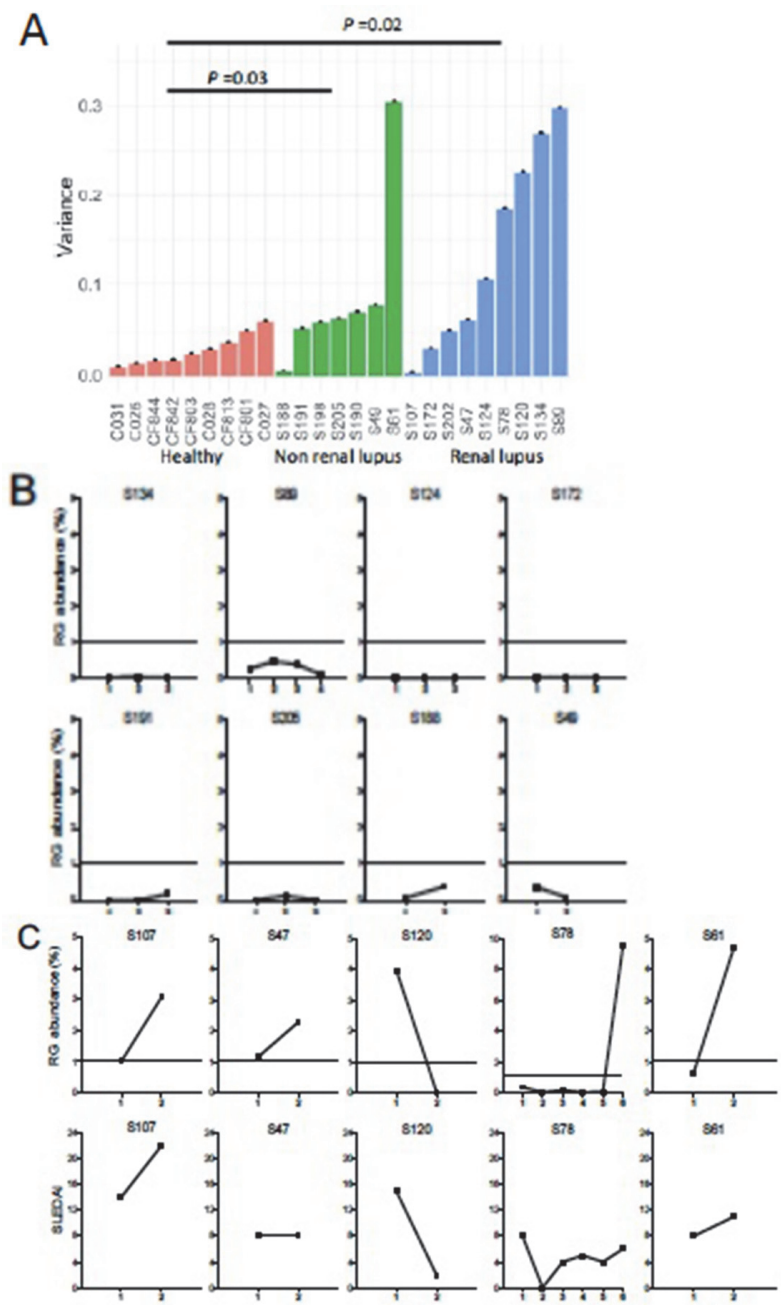

Abstract 511 Figure 1 Lupus patients have more unstable gut microbiota than healthy individuals, and a subset of Lupus patients have blooms of Ruminococcus blautia gnavus concordant with disease flares. (A) To compare the overall dynamics of shifts in fecal communities sampled overtime in different subjects, subject variances were computed based on Jensen-Shannon Divergence using the Tw2 statistic. Variances in these three groups were significantly different (Kruskal Wallis ANOVA, $p=0.03$ ). Patients with Lupus nephritis, based on ACR criteria, whereas the patients in the non-renal group were without a history of documented lupus nephritis. Temporal-dependent variance documents instability in the gut microbiota communities of Lupus patients compared to healthy. (B) In 11 SLE patients, a stable low abundance of RG representation was detected. (C) In 5/16 (31\%) of SLE patients evaluated overtime, abundance of RG fluctuated greatly overtime. In these cases, RG abundance at much higher levels were present in fecal samples obtained proximal to visits in which disease flares were documented. In all but one excluded of these patients had documented LN. RG relative abundance was evaluated for 16 SLE in 44 samples obtained at different time points, and for CTL subjects in 49 samples obtained at different time points, which ranged from 2-12 samples per donor. Dotted line depicts $1 \%$ threshold of $16 \mathrm{~S}$ rRNA amplicon representing RG abundance. Note that in panel C, for patient S78 the greater range of RG abundance necessitated a different scale. SLEDAI $\geq 8$ was considered high disease activity. 
recent in vivo pathogenesis studies in mice, have shown that intestinal colonization by RG strains isolated from Lupus patients in flare, but not RG strains from healthy adults, induce zonulin-dependent increases in intestinal permeability, RG translocation to mesenteric lymph nodes as well as serum IgG anti-RG antibody and anti-native DNA autoantibody responses.

Conclusions As many Lupus patients are known to suffer relapsing illness despite appropriate treatment, we speculate that gut blooms of pathogenic bacteria that impair gut barrier function and stoke systemic inflammation directly contribute to immunopathogenesis. We propose that future therapeutic interventions designed to promote a durable remission consider the potential necessity to target both the immunologic abnormalities of the disease, as well as re-establish stability within the gut microbiota community.

Acknowledgments This work was supported in part by National Institutes of Health Grants; R01-AR42455, P50 AR070591, the Lupus Research Alliance, and the Judith and Stewart Colton Autoimmunity Center (GJS). 16S rRNA amplicon sequence determinations and analysis were supported by the P. Robert Majumder Charitable Trust.

\section{THE RISING INCIDENCE, PREVALENCE AND MORTALITY GAP OF LUPUS NEPHRITIS: A POPULATION-BASED STUDY OVER FOUR DECADES}

\begin{abstract}
1,2Mehmet Hocaoglu, 'Maria 0 Valenzuela-Almada, 'Jesse Y Dabit, 'Shirley-Ann OseiOnomah, ${ }^{1,3}$ Baptiste Chevet, ${ }^{4}$ Rachel E Giblon, ${ }^{1,4}$ Cynthia S Crowson, ${ }^{1,5}$ Alí Duarte-García*. ${ }^{1}$ Division of Rheumatology, Mayo Clinic, Rochester, MN, USA; ${ }^{2}$ Department of Medicine, University of Maryland Medical Center, Midtown Campus, Baltimore, MD, USA; ${ }^{3}$ Division of Rheumatology, Brest Teaching Hospital, Brest, France; LBAI, UMR1227, Univ Brest, Inserm, CHU de Brest, Brest, France; ${ }^{4}$ Department of Quantitative Health Sciences, Mayo Clinic, Rochester, MN, USA; ${ }^{5}$ Division of Nephrology and Hypertension, Mayo Clinic, Rochester, MN, USA
\end{abstract}

\subsection{6/lupus-2021-lupus21century.30}

Background There is paucity of population-based studies that investigate the epidemiology of lupus nephritis (LN) and its long-term outcomes.

Methods We used the Rochester Epidemiology Project (REP) a record-linkage system that includes all residents from Olmsted County, Minnesota since 1966 with additional counties since 2010. A cohort of LN patients between 1976 and 2018 in an eight-county region was identified based on (1) biopsy proven LN in the presence of a positive ANA or ds-DNA antibody, or (2) meeting ACR/EULAR 2019 criteria and having documented proteinuria $(500 \mathrm{mg}$ in $24 \mathrm{hrs}$ urine collection or protein/creatinine ratio $>0.5)$ that did not have a better explanation than SLE. Age- and sex-specific incidence rates and point prevalence for four decades, adjusted to 2000 US total population, were reported. Standardized mortality ratio (SMR), survival rates, and time to end-stage renal disease (ESRD) were estimated using Kaplan-Meier methods. Poisson regression models were used to look at trends in relative survival over time.

Results There were 72 incident LN from 1976-2018. Mean age was 38.4 years (SD 16.24), 76\% were female, and 69\% non-Hispanic White. The overall LN incidence between 1976 and 2018 was estimated at 1 per 100,000 (95\%CI 0.8-1.3). The incidence was highest in 30-39 age group. The overall incidence of $\mathrm{LN}$ increased from 0.7 to 1.3 with sex specific incidence in males quadrupling from 0.2 to 0.8 per 100,000 between 1976-1988 and 2000-2018 periods (table 1). LN prevalence increased from 16.8 per 100,000 in 1985 to 21.2 in 2015. LN had SMR of 6.33 (95\%CI 3.81-9.89) compared to the general population. Survival was $50 \%$ at 20 years, and among survivors $40 \%$ had ESRD. There was a widening mortality gap with an increase in the SMR of $7 \%$ per year $(1.07$; 95\%CI 1.03-1.10, $\mathrm{p}<0.001)$ or almost a doubling of the SMR every 10 years (1.90 per 10 years; 95\%CI 1.33-2.70). Conclusions The incidence and prevalence of $\mathrm{LN}$ has increased in the last 40 years. Long-term outcomes in patients with LN remain poor. Patients with $\mathrm{LN}$ had a mortality rate of more than $50 \%$ at 20 years and of those surviving, $40 \%$ had ESRD. The widening mortality gap we observed with near doubling of SMR each decade shows that the mortality reduction in LN patients is not keeping up with the mortality improvements of the general population despite changes in clinical practice over the decades.

\section{RESPONSE GENE TO COMPLEMENT -32 FACILITATES LOCAL RECRUITMENT OF IL-17- PRODUCING CELLS IN IMMUNE COMPLEX MEDIATED GLOMERULONEPHRITIS THROUGH THE CCR6/CCL2O AXIS}

\footnotetext{
1,2Violeta Rus ${ }^{*},{ }^{1}$ Alexandru Tatomir, ${ }^{1,2}$ Vinh Nguyen, ${ }^{1} J o h n$ Papadimitriou, ${ }^{1}$ Sergei Atamas, 1,2 Irina G Luzina, ${ }^{3}$ Tudor Badea, ${ }^{4}$ Sonia Vlaicu, ${ }^{1,2}{ }^{1}$ Horea Rus. ${ }^{1}$ University of Maryland School of Medicine, Baltimore, MD, USA; ${ }^{2}$ VA Maryland Health Care System, Baltimore, MD, USA; ${ }^{3} \mathrm{NIH}$, NEI, Bethesda, MD, USA; ${ }^{4}$ University of Medicine and Pharmacy 'Iuliu Hatieganu', Cluj-Napoca, Romania
}

10.1136/lupus-2021-lupus21century.31

Background Response Gene to Complement (RGC)-32 is a cell cycle regulator induced by complement activation, growth factors and cytokines. RGC-32 mediates TGF- $\beta$ dependent profibrotic pathways, while in immune cells promotes the

Abstract 512 Table 1 Age-adjusted incidence (1976-2018) and prevalence $(1985,1995,2005,2015)$ rates of Lupus Nephritis (LN), 8-county region in Southeast Minnesota.

\begin{tabular}{|c|c|c|c|c|c|c|c|c|c|}
\hline & \multicolumn{5}{|c|}{ LN incidence rates $/ 100,000(95 \% \mathrm{Cl})$ * } & \multicolumn{4}{|c|}{ LN Prevalence rates $/ 100,000(95 \% \mathrm{Cl})$ * } \\
\hline & 1976-1989 & 1990-1999 & $2000-2009$ & $2010-2018$ & $1976-2018$ & $01 / 01 / 1985$ & $01 / 01 / 1995$ & $01 / 01 / 2005$ & $01 / 01 / 2015$ \\
\hline Females & $1.2(0.4,2.0)$ & $1.3(0.4,2.2)$ & $1.7(0.7,2.7)$ & $1.7(1.0,2.4)$ & $1.5(1.1,1.9)$ & $25.9(11.4,40.5)$ & $30.7(16.7,44.7)$ & $36.5(22.4,50.7)$ & $33.4(24.7,42.1)$ \\
\hline Males & $0.2(0.0,0.6)$ & $0.0(0.0,0.0)$ & $0.6(0.0,1.2)$ & $0.8(0.4,1.3)$ & $0.5(0.3,0.8)$ & $7.1(0.0,15.1)$ & $6(0.0,13)$ & $1.4(0.0,4.3)$ & $8.6(4.1,13)$ \\
\hline Overall & $0.7(0.2,1.2)$ & $0.6(0.2,1.1)$ & $1.2(0.6,1.8)$ & $1.3(0.9,1.7)$ & $1.0(0.8,1.3)$ & $16.8(8.3,25.3)$ & $18.5(10.7,26.4)$ & $19.4(12.1,26.8)$ & $21.2(16.3,26.2)$ \\
\hline
\end{tabular}

